\title{
Quantum conditional cloning of continuous variable entangled states
}

K. Liu

liukui@sxu.edu.cn

\section{J. R. Gao}

State Key Laboratory of Quantum Optics and Quantum Optics Devices, Institute of Opto-Electronics, Shanxi University, Taiyuan 030006, Peoples Republic of China

State Key Laboratory of Quantum Optics and Quantum Optics Devices, Institute of Opto-Electronics, Shanxi University, Taiyuan 030006, Peoples Republic of China

We extend the technique of conditional preparation to a quantum cloning machine, and present a protocol of $1 \rightarrow 2$ conditional cloning of squeezed state and entanglement states. It is shown that the entanglement degree of the cloned entangled states can be well preserved even when the fidelity between the input and output states is beyond the limit of $4 / 9$. This scheme is practicable since only the linear elements of beam splitters, homodyne detections, optical modulations and electrical trigger system, are involved.

[DOI: http://dx.doi.org/10.2971/jeos.2014.14057]

Keywords: Quantum cloning, conditional selection, squeezed state, entanglement

\section{INTRODUCTION}

Quantum cloning is a fundamental issue in quantum information processing and the topic of quantum cloning is concerned with devising a quantum cloning machine to make optimal approximate copies [1]-[3]. The quantum cloning machine was firstly developed for quantum discrete variables (DV) or qubits [2,3], and later it was extended to quantum continuous variables (CV) [4]. The implementations of $\mathrm{CV}$ cloning machines using parametric amplifiers and beam splitters were proposed in 2004 [6, 7]. Quantum cloning for CV has attracted more and more interest [8]-[11], especially the quantum cloning of CV entangled states. Quantum entanglement [12]-[14] is a central resource in quantum information processes such as quantum teleportation [15], quantum dense code [16], and quantum cryptography protocols [17]. A CV entanglement cloning machine can be used in quantum information processing, such as error correction in the quantum computation [18] and quantum eavesdropping [19] in quantum cryptography. The cloning of CV entangled states has been presented [20], but it is necessary to involve 6 extra squeeze and unsqueeze gates.

Conditional preparation is an important technique in quantum information experiments. Recent developments in both theory and experiment demonstrate that a nonclassical quantum state of light can be conditional prepared in the continuous variable regime [21, 22]. With this technique of conditional preparation, the purification of squeezed and entangled states for CV was experimentally accomplished [23, 24]. Using a similar technique of conditional post-selection, the entanglement distillation of mesoscopic quantum states was obtained experimentally [25], and the entanglement distillation of Gaussian input states via conditional subtraction of photons from input states was also realized [26].
In this paper, we extend the technique of conditional preparation to a quantum cloning machine, and present a protocol of $1 \rightarrow 2$ conditional cloning of squeezed state and entangled states. The entanglement degree of each of the two cloned entangled states can be well preserved if suitable correlation thresholds of the corresponding quadrature components of the input entangled state are chosen. Meanwhile, the fidelity between the input and cloned entangled state is also discussed. It shows that the fidelity can also be optimized via conditional preparation. The conditional quantum cloning machine can be applied into quantum information processing.

\section{CONDITIONAL CLONING OF SINGLE-MODE SQUEEZED STATE}

Figure 1 shows the scheme for the conditional cloning machine of an arbitrary Gaussian state. At the input side of the cloning machine, an unknown quantum state (represented by the annihilation operator $\hat{a}_{1}$ ) is divided into two parts $\hat{a}_{3,4}=\left(\hat{a}_{1} \pm \hat{a}_{2}\right) / \sqrt{2}$ by a $50 / 50$ beam splitter BS1, where $\hat{a}_{2}$ is the vacuum mode in the other input channel of BS1. The output state $a_{4}$ is coupled with vacuum states $a_{5}$ at a 50/50 beam splitter BS2, the amplitude $\hat{x}_{6}=\operatorname{Re}\left[\left(\hat{a}_{4}+\hat{a}_{5}\right) / \sqrt{2}\right]$ and the phase $p_{7}=\operatorname{Im}\left[\left(a_{4}-a_{5}\right) / \sqrt{2}\right]$ quadratures of the two output mode $\left(\hat{a}_{6,7}\right)$ of BS2 are simultaneously measured using two ideal balanced homodyne detections (BHDs). The mode of $\hat{a}_{3}$ is displaced by the measurement $\hat{x}_{6}, \hat{p}_{7}$ with gain factor $g$, $\hat{a}_{3} \rightarrow \hat{a}_{8}=\hat{a}_{3}+g\left(\hat{x}_{6}+i \hat{p}_{7}\right)$. In order to get the optimal cloning states, we must keep the unity cloning gains, in other words, the expectations of output state must be equal to the expectations of input state $\left(\left\langle\hat{x}_{\text {in }}\right\rangle=\left\langle\hat{x}_{\text {clone }}\right\rangle,\left\langle\hat{p}_{\text {in }}\right\rangle=\left\langle\hat{p}_{\text {clone }}\right\rangle\right)$ [10], the gain factor $g$ is thus taken to be $2^{1 / 2}$. Then, the values of amplitude and phase quadratures $(\hat{x}=\operatorname{Re}(\hat{a})$ and $\hat{p}=\operatorname{Im}(\hat{a}))$ of 


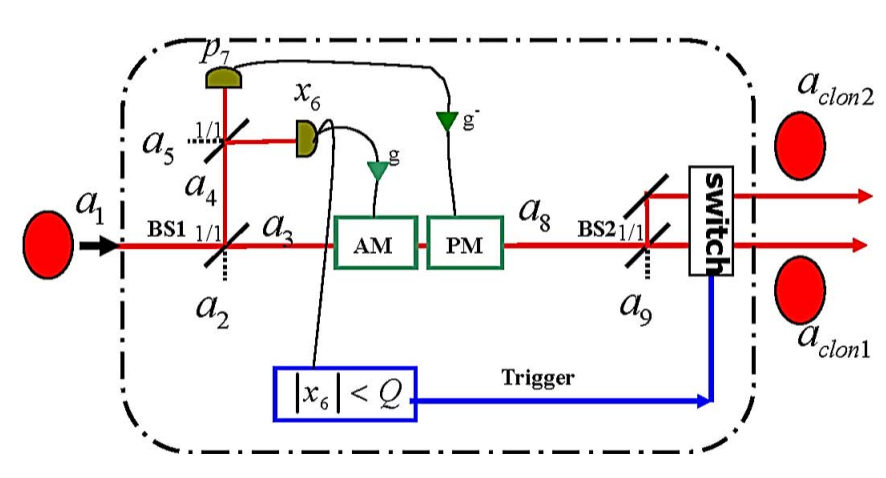

FIG. 1 (Color online) Scheme for the conditional cloning machine of an arbitrary Gaussian state. The machine is the symmetric $1 \rightarrow 2$ linear quantum cloning machine. $a_{1}$ : the input arbitrary state; $a_{\text {clon } 1}$ it $a_{\text {clon2 }}$ : clones of the input state; AM: amplitude modulator; PM: phase modulator; $\mathrm{BS}_{1}, \mathrm{BS}_{2}, \mathrm{BS}_{3}(1 / 1): 50 / 50$ beam splitter; $\left|\Delta x_{6}\right|<Q$ : the condition taken as the trigger; Switch: turn on or turn off.

mode $\hat{a}_{8}$ are: $x_{8}=x_{3}+\sqrt{2} x_{6}$ and $p_{8}=p_{3}+\sqrt{2} p_{7}$. At last, the mode is divided into two cloning states $\hat{a}_{\text {clon } 1}=\left(\hat{a}_{8}+\hat{a}_{9}\right) / \sqrt{2}$ and $\hat{a}_{\text {clon } 2}=\left(\hat{a}_{8}-\hat{a}_{9}\right) / \sqrt{2}$ by a beam splitter BS3, where $\hat{a}_{9}$ is the vacuum state induced by the beam splitter. For a squeezed state input, the key point of our supposed clone machine is the conditional selection of the measurement value of the variance of squeezed component. We consider a set of amplitude quadrature squeezed state inputs with unknown squeezing and expectation and set a certain trigger threshold $Q$ for $\Delta x_{6}$, which is the permitted maximum fluctuation of the squeezed quadrature component, where the cloning states are only prepared when $\left|\Delta x_{6}\right|<Q$.

For a Gaussian input state, the Wigner function is:

$$
W(\alpha)=\frac{1}{2 \pi \sqrt{V_{x} V_{p}}} \exp \left[-\frac{(x-\bar{x})^{2}}{2 V_{x}}-\frac{(p-\bar{p})^{2}}{2 V_{p}}\right],
$$

where $\alpha=x+i y . \bar{x}$ and $\bar{p}$ are the expectations of the amplitude and phase quadratures and $V_{x}$ and $V_{p}$ are the variances of the amplitude and phase quadratures, respectively; when $V_{x}=V_{p}=1 / 4$, the state is the coherent state; when $V_{x}$ or $V_{p}<1 / 4$, the state is the amplitude or phase squeezed state.

The overall Wigner distribution of output modes (after beam splitter 2) can be transformed from the initial Wigner distribution $W\left(\alpha_{1}\right) \cdot W\left(\alpha_{2}\right) \cdot W\left(\alpha_{5}\right) \cdot W\left(\alpha_{9}\right)$ into:

$$
\begin{aligned}
& W\left(\alpha_{\text {clon } 1}, \alpha_{\text {clon } 2}, \alpha_{6}, \alpha_{7}\right) 1 \\
&= \frac{1}{(2 \pi)^{4} \sqrt{V_{x 1} V_{p 1} V_{x 2} V_{p 2} V_{x 5} V_{p 5} V_{x 9} V_{p 9}}} \\
& \cdot \exp \left[-\frac{\left(x_{\text {clon } 1}+x_{\text {clon } 2}-x_{6}+x_{7}-\bar{x}\right)^{2}}{8 V_{x 1}}\right] \\
& \cdot \exp \left[-\frac{\left(p_{\text {clon } 1}+p_{\text {clon } 2}-p_{7}+p_{6}-\bar{p}\right)^{2}}{8 V_{p 1}}\right] \\
& \cdot \exp \left[-\frac{\left(x_{\text {clon } 1}+x_{\text {clon } 2}-3 x_{6}-x_{7}\right)^{2}}{8 V_{x 2}}\right] \\
& \cdot \exp \left[-\frac{\left(p_{\text {clon } 1}+p_{\text {clon } 2}-3 p_{7}+p_{6}\right)^{2}}{8 V_{p 2}}\right] \\
& \cdot \exp \left[-\frac{\left(x_{6}-x_{7}\right)^{2}}{\left.4 V_{x 5}-\frac{\left(p_{6}-p_{7}\right)^{2}}{4 V_{p 5}}\right]}\right] \\
& \cdot \exp \left[-\frac{\left(x_{\text {clon } 1}-x_{\text {clon } 2}\right)^{2}}{4 V_{x 9}}-\frac{\left(p_{\text {clon } 1}-p_{\text {clon } 2}\right)^{2}}{4 V_{p 9}}\right]
\end{aligned}
$$

The two cloned output states $\left(a_{\text {clon } 1}, a_{\text {clon } 2}\right)$ have the same properties, and we can only consider the cloning state $a_{\text {clon } 1}$ in the cloning process. The joint distribution for mixture states $x_{6}$ with $x_{\text {clon } 1}$ can be calculated by integrating $W\left(\alpha_{\text {clon } 1}, \alpha_{\text {clon } 2}, \alpha_{6}, \alpha_{7}\right)$ over other modes.

$$
\begin{aligned}
& W_{\text {out }}\left(x_{\text {clon } 1}, x_{6}\right) \\
& =\int_{-\infty}^{\infty} \int_{-\infty}^{\infty} \int_{-\infty}^{\infty} \int_{-\infty}^{\infty} \int_{-\infty}^{\infty} \int_{-\infty}^{\infty} W d x_{\text {clon } 2} d x_{7} d p_{\text {clon } 1} d p_{\text {clon } 2} d p_{6} d p_{7} \\
& =\frac{4}{\pi \sqrt{2\left(1+4 V_{x 1}\right)}} \exp \left[-\frac{3+4 V_{x 1}}{1+4 V_{x 1}}\left(x_{\text {clon } 1}-\bar{x}\right)^{2}\right] \\
& =\frac{4}{\pi \sqrt{2\left(1+4 V_{x 1}\right)}} \exp \left[-\frac{3+4 V_{x 1}}{1+4 V_{x 1}}\left(x_{\text {clon } 1}-\bar{x}\right)^{2}\right] \\
& \left.\cdot \exp \left[-4 \Delta x_{6}^{2}+\frac{\bar{x}}{2}\right)^{2}+4\left(x_{\text {clon } 1}-\bar{x}\right)\left(x_{6}-\frac{\bar{x}}{2}\right)\right]
\end{aligned}
$$

For the clone machine discussed here, the conditional selection of $x_{6}$ is done to improve the fidelity of the cloning state. If the fluctuation of measured amplitude quadrature falls below a certain trigger threshold $Q$ $\left(\left|x_{6}-\bar{x}_{6}\right|=\left|x_{6}-\frac{\bar{x}}{2}\right|=\left|\Delta x_{6}\right|<Q\right)$, we switch on the process to reconstruct the output state, otherwise, the reconstruction is discarded. In the experiment, we can get $\Delta x_{6}$ by using a signal processing system [21, 23], where $\Delta x_{6}$ corresponds to the AC part of the measured data. The probability distribution of the amplitude quadrature of output cloning state is given by:

$$
W_{\text {out }}\left(x_{\text {clon } 1}\right)=\frac{1}{P} \int_{Q}^{Q} W_{\text {out }}\left(x_{\left(\operatorname{clon} 1, x_{6}\right)} d \Delta x_{6} .\right.
$$

Here the normalization factor $P$ denotes the probability of positive trigger events from BHD, called probability of success of cloning.

$$
P=\int_{-Q}^{Q} \int_{-\infty}^{\infty} W\left(x_{\left(\operatorname{clon} 1, x_{6}\right)} d \Delta x_{6} d x_{\text {clon } 1}\right.
$$

It can be easily seen that the expectation value of the clone is

$$
\bar{x}_{\text {clon } 1}=\int_{-\infty}^{\infty} W_{\text {out }}\left(x_{\text {clon } 1}\right) x_{c l o n 1} d x_{\text {clon } 1}=\bar{x}
$$

Regardless of the trigger threshold $Q$, the expectations of the output states always are equal to the expectations of the input states. Therefore, we can neglect the expectation values of the cloning states, and only consider the fluctuations of the cloning states.

The squeezing of the clone (here, amplitude quadrature squeezing) is most conveniently characterized by the variance of its quadrature $x_{\text {clon } 1}$

$$
V_{\text {out }}\left(x_{\text {clon } 1}\right)=\int_{-\infty}^{\infty} W_{\text {out }}\left(x_{\text {clon } 1}\right)\left(x_{\text {clon } 1}-\bar{x}\right)^{2} d x_{\text {clon } 1}
$$

There is no limit to the phase quadrature $p_{7}$, so the distribution of the phase quadrature of output cloning state is directly 
given by:

$$
\begin{aligned}
W_{\text {out }}\left(p_{\text {clon } 1}\right) & \int_{-\infty}^{\infty} \int_{-\infty}^{\infty} \int_{-\infty}^{\infty} \int_{-\infty}^{\infty} \int_{-\infty}^{\infty} \int_{-\infty}^{\infty} \int_{-\infty}^{\infty} W \\
& \cdot d p_{\text {clon } 2} d p_{6} d p_{7} d x_{\text {clon } 1} d x_{\text {clon } 2} d x_{6} d x_{7} \\
= & \frac{1}{\sqrt{\frac{\pi}{2}\left(1+4 V_{p 1}\right)}} \exp \left[-\frac{2\left(p_{\text {clon } 1}-\bar{p}\right)^{2}}{1+4 V_{p 1}}\right]
\end{aligned}
$$

The final Wigner function of cloning state can be expressed as:

$$
W_{\text {clon }}\left(\alpha_{\text {clon } 1}\right)=W_{\text {out }}\left(x_{\text {clon } 1}\right) \cdot W_{\text {out }}\left(p_{\text {clon } 1}\right)
$$

Fidelity is represented by the overlap between the Wigner functions of the original and the output states [27], so the fidelity of the cloning squeezed state is

$$
F=\pi \int W(\alpha) W_{\text {clon }}(\alpha) d^{2} \alpha
$$

The results of numerical calculations are shown in Figure 2. The dependence of variance of the output squeezed state $V_{\text {out }}$, the fidelity $F$ and the successful cloning probability $P$ on the selection threshold are described in Figure 2(a), 2(b) and 2 (c) respectively, and three squeezing degrees $(2.2 \mathrm{~dB}, 5.2 \mathrm{~dB}$, $10 \mathrm{~dB}$ ) of the input squeezed states are considered in the figures. Due to the fragile nature of squeezing with loss, we can find that the lower squeezing degree of the input state is, the higher fidelity of the cloning is [28], that is, the higher squeezing is more fragile and decays more easily. By the conditional selection to the states, some extra noise, which is derived from the cloning process, can be eliminated. As a result, for the fixed input squeezed state, the better the fidelity is, the lower the threshold is, but it is inevitable to sacrifice the probability of success. When the $Q$ is above 2, which is eight times normalized to the shot noise limit, the conditional cloning machine tends towards the general cloning machine [9]. By the conditional cloning machine, the squeezed state can be duplicated with high fidelity and with low threshold, but it cannot be duplicated perfectly, because the vacuum noise $a_{9}$, which is coupled into the final cloning states by BS3, cannot be eliminated by our scheme.

Furthermore, if the input of the cloning machine is a phase squeezed state, the select operation should be taken on the phase quadrature $\left(\left|\Delta p_{7}\right|<Q\right)$, and we also can get good quality clones with high fidelity and squeezing.

\section{CONDITIONAL CLONING OF ENTANGLED STATES}

Now, let us consider entangled states conditional cloning, for which the proposed setup is depicted in Figure 3. We consider a set of entangled states with unknown entanglement and expectation. The input entangled states are expressed as [29]:

$$
\begin{aligned}
& W_{\text {epr }}\left(\alpha_{1}, \alpha_{11}\right) \\
& =\frac{4}{\pi^{2}} \exp \left[-\frac{\left(\Delta x_{1}+\Delta x_{11}\right)^{2}+\left(\Delta p_{1}-\Delta p_{11}\right)^{2}}{e^{-2 r}}\right] \\
& \cdot \exp \left[-\frac{\left(\Delta x_{1}+\Delta x_{11}\right)^{2}+\left(\Delta p_{1}+\Delta p_{11}\right)^{2}}{e^{2 r}}\right],
\end{aligned}
$$
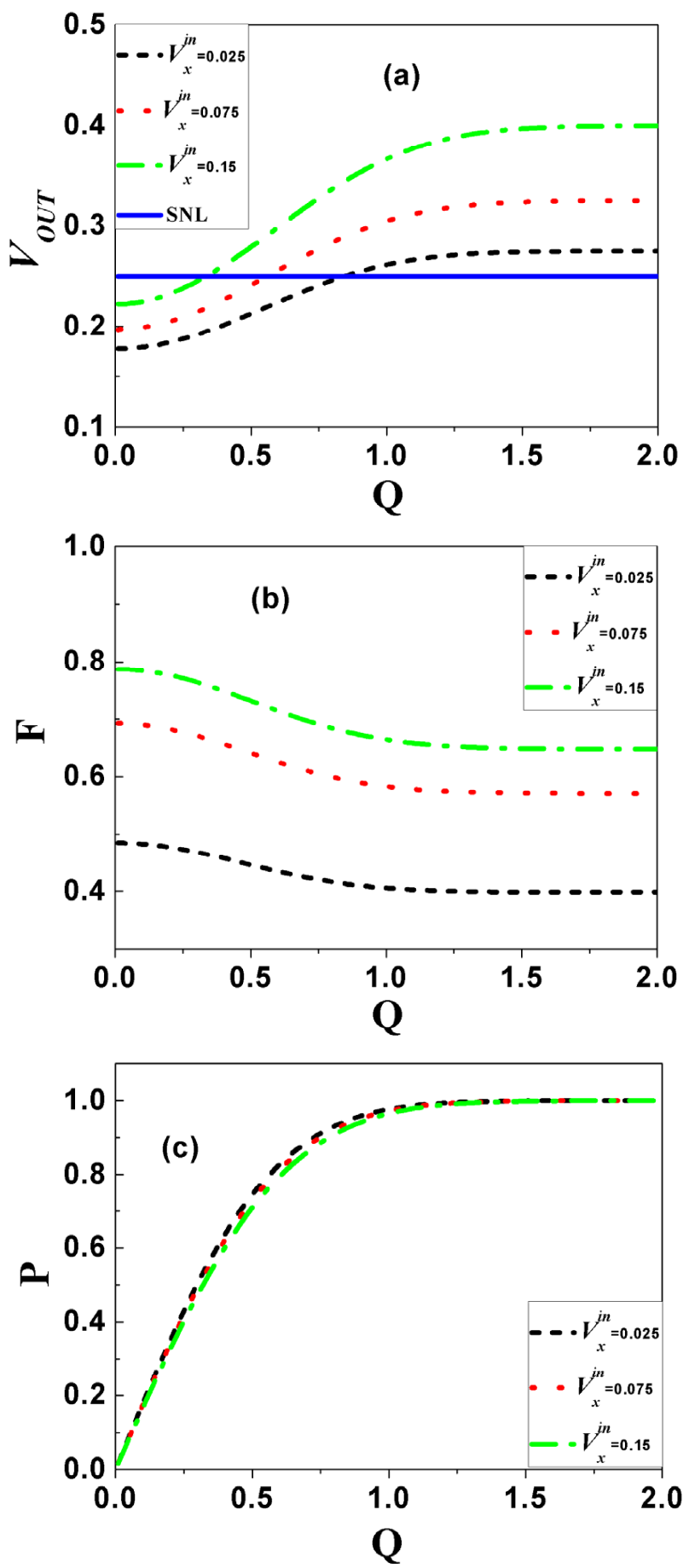

FIG. 2 Results of amplitude squeezed states conditional cloning with different threshold value. (a) The amplitude variance of clone $V_{\text {out }}$ depending on threshold value $Q$. (b) The fidelity of clone $F$ depending on threshold value $Q$. (c) The probability of success of clone $P$ depending on threshold value $Q$. We consider three input states with different degree of amplitude squeezing: $V_{x}^{\text {in }}=0.15$, corresponding to $2.2 \mathrm{~dB}$ squeezing (green dash dot line), $v_{x}^{i n}=0.075$, corresponding to $5.2 \mathrm{~dB}$ squeezing (red dot line), and $V_{x}^{i n}=0.025$, corresponding to $10 \mathrm{~dB}$ squeezing (black dash line). The shot noise limitation (SNL) is $V=0.25$ (blue solid line).

where $\Delta x_{i}=x_{i}-\bar{x}_{i}$ and $\Delta p_{i}=p_{i}-\bar{p}_{i}(i=1,11)$, and $r$ represents the parameter of squeezing.

The entangled states exhibit the two commuting quadratures $x_{+}=\left(x_{1}+x_{11}\right) / \sqrt{2}$ and $p_{-}=\left(p_{1}-p_{11}\right) / \sqrt{2}$ of two squeezed states, and can be quantified by the EinsteinPodolsky-Rosen (EPR) uncertainty $\Delta_{E P R}=V\left(x_{+}\right)+V\left(p_{-}\right)$. The states will be entangled if $\Delta_{E P R}<1 / 2$ [30]. 


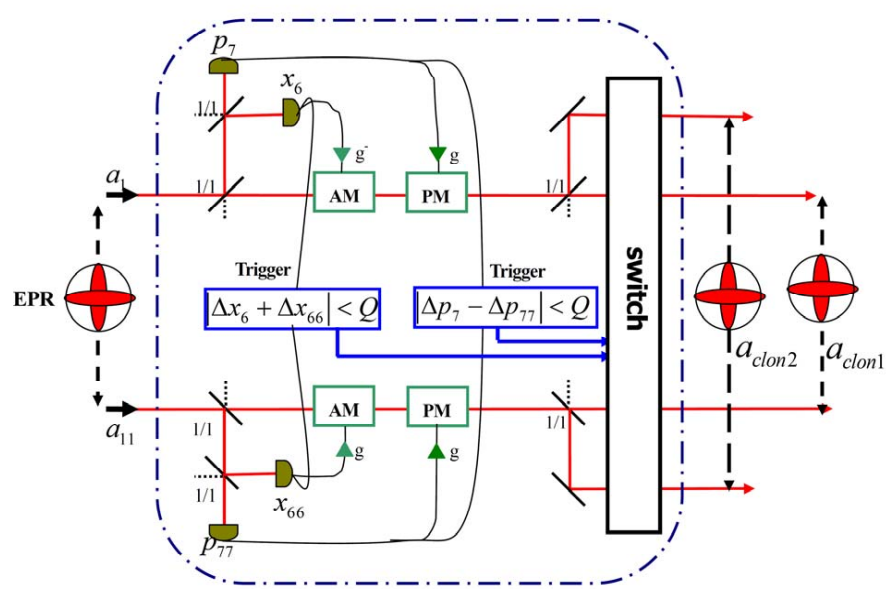

FIG. 3 (Color online) Scheme for conditional cloning machine of entangled states. The machine is the symmetric $1 \rightarrow 2$ linear quantum cloning machine. $a_{1}$ c $a_{11}$ : the input entangled states; $a_{\text {clon } 1}$ at $a_{\text {clon2 }}$ : clones of entangled states and the dashed black lines indicate which modes are entangled; AM: amplitude modulator; PM: phase modulator; 1/1: 50/50 beam splitter; $\left|\Delta x_{6}+\Delta x_{66}\right|<Q$ at $\left|\Delta p_{7}-\Delta p_{77}\right|<Q$ : the conditions taken as the triggers of switch. Switch: turn on/turn off.

The implementation of entangled states conditional cloning is similar to the local cloning of entangled states [20]. The selected threshold is added for a pair of correlated quadratures fluctuation here, this is corresponding to the entanglement of the input state. Only if both conditions $\left|\Delta x_{Q}\right|=\left|\Delta x_{6}+\Delta x_{66}\right|<Q$ and $\left|\Delta p_{Q}\right|=\left|\Delta p_{7}-\Delta p_{77}\right|<Q$ are satisfied, can the clones of the entangled states be prepared.

The input entangled states can be rewritten as two singlemode squeezed states $\bar{a}_{+}$and $\bar{a}_{-}$for simplification,

$$
\begin{aligned}
& W_{\operatorname{erp}}\left(\alpha_{1}, \alpha_{1} 1\right)=W_{+}\left(\alpha_{+}\right) \cdot W_{-}\left(\alpha_{-}\right) \\
& W_{+}\left(\alpha_{+}\right)=\frac{2}{\pi} \exp \left[-2 e^{2 r} x_{+}^{2}-2 e^{-2 r} p_{+}^{2}\right] \\
& =\frac{1}{2 \pi \sqrt{V_{x+} V_{p+}}} \exp \left[-\frac{\Delta x_{+}^{2}}{2 V_{x+}}-\frac{\Delta p_{+}^{2}}{2 V_{p+}}\right] \\
& W_{-}\left(\alpha_{-}\right)=\frac{2}{\pi} \exp \left[-2 e^{-2 r} x_{-}^{2}-2 e^{2 r} p_{-}^{2}\right] \\
& =\frac{1}{2 \pi \sqrt{V_{x-} V_{p-}}} \exp \left[-\frac{\Delta x_{+}^{2}}{2 V_{x-}}-\frac{\Delta p_{+}^{2}}{2 V_{p-}}\right]
\end{aligned}
$$

Here $\alpha_{ \pm}=\left(\alpha_{1} \pm \alpha_{11}\right) / \sqrt{2}, \Delta x_{ \pm}=\left(\Delta x_{1} \pm \Delta x_{11}\right) / \sqrt{2}$, $\Delta p_{ \pm}=\left(\Delta p_{1} \pm \Delta p_{11}\right) / \sqrt{2}, V_{x+}=V_{p-}=1 / 4 \cdot \exp (-2 r)$, and $V_{x-}=V_{p+}=1 / 4 \cdot \exp (2 r)$.

In fact the entangled states can be transformed into two singlemode squeezed states as Eq. (12), which represent entangled compound states and where $r$ represents parameter of squeezing; therefore we can treat the entangled states conditional cloning, shown in Figure 3, as the conditional cloning of two independent single-mode squeezed states, and it is analogous to the method in ref. [26, 31]. According to the discussion in part 2, we can easily get the expression of the Wigner function $W_{+}\left(\alpha_{+ \text {clon }}\right), W_{-}\left(\alpha_{- \text {clon }}\right)$ of cloning state of $\bar{a}_{+}$and $\bar{a}_{-}$, respectively.

$$
\begin{aligned}
& W_{+}\left(\alpha_{+ \text {clon }}\right)=W_{+}\left(x_{+ \text {clon }}\right) \cdot W_{+}\left(p_{+ \text {clon }}\right) \\
& W_{-}\left(\alpha_{- \text {clon }}\right)=W_{-}\left(x_{- \text {clon }}\right) \cdot W_{-}\left(p_{- \text {clon }}\right)
\end{aligned}
$$

$$
\begin{aligned}
W_{+}\left(x_{+ \text {clon }}\right)= & \frac{1}{P_{+}} \int_{-Q}^{Q} \frac{4}{\pi \sqrt{2\left(1+4 V_{x+1}\right)}} \\
& \cdot \exp \left[-\frac{3+4 V_{x+}}{1+4 V_{x+}} \Delta x_{+ \text {clon }}^{2}\right] \\
& \cdot \exp \left[-4 \Delta x_{Q}^{2}+4 \Delta x_{+ \text {clon }} \Delta x_{Q}\right] d \Delta x_{Q}
\end{aligned}
$$

$$
\begin{aligned}
P_{+}= & \int_{-Q}^{Q} \int_{-\infty}^{\infty} \frac{4}{\pi \sqrt{2\left(1+4 V_{x+}\right)}} \\
& \cdot \exp \left[-\frac{3+4 V_{x+}}{1+4 V_{x+}} \Delta x_{+ \text {clon }}^{2}\right] \\
& \cdot \exp \left[-4 \Delta x_{Q}^{2}+4 \Delta x_{+ \text {clon }} \Delta x_{Q}\right] d \Delta x_{Q} d x_{\text {clon } 1}
\end{aligned}
$$

$$
W_{+}\left(p_{+ \text {clon }}\right)=\frac{1}{\sqrt{\frac{\pi}{2}\left(1+4 V_{p+}\right)}} \exp \left[-\frac{2 \Delta p_{+ \text {clon }}^{2}}{1+4 V_{p+}}\right]
$$

$$
\begin{aligned}
& W_{+}\left(x_{- \text {clon }}\right)=\frac{1}{\sqrt{\frac{\pi}{2}\left(1+4 V_{x-}\right)}} \exp \left[-\frac{2 \Delta x_{- \text {clon }}^{2}}{1+4 V_{x+}}\right] \\
& W_{-}\left(p_{- \text {clon }}\right)=\frac{1}{P_{-}} \int_{-Q}^{Q} \frac{4}{\pi\left(1+4 V_{p-}\right.} \\
& \cdot \exp \left[-\frac{3+4 V_{p-}}{1+4 V_{p-}} \Delta p_{- \text {clon }}^{2}-4 \Delta p_{Q}^{2}\right] \\
& \cdot \exp \left[+4 \Delta p_{-c l o n} \Delta p_{Q}\right] d \Delta p_{Q}
\end{aligned}
$$

$$
\begin{aligned}
& P_{-}= \int_{-Q}^{Q} \int_{-\infty}^{\infty} \frac{4}{\pi \sqrt{2\left(1+4 V_{p-}\right)}} \\
& \cdot \exp {\left[-\frac{3+4 V_{p-}}{1+4 V_{p-}} \Delta p_{-c l o n}^{2}\right] } \\
& \cdot \exp \left[-4 \Delta p_{Q}^{2}+4 \Delta p_{- \text {clon }} \Delta p_{Q}\right] d \Delta p_{Q} d p_{\text {clon } 1}
\end{aligned}
$$

The squeezing of cloning states can be calculated from Eq. (7).

$$
\begin{aligned}
& V_{\text {out }}\left(x_{+ \text {clon }}\right)=\int_{-\infty}^{\infty} W\left(x_{+ \text {clon }}\right) \Delta x_{+ \text {clon }}^{2} d x_{+ \text {clon }} \\
& V_{\text {out }}\left(p_{- \text {clon }}\right)=\int_{-\infty}^{\infty} W\left(p_{- \text {clon }}\right) \Delta p_{- \text {clon }}^{2} d p_{- \text {clon }}
\end{aligned}
$$

The entanglement of the cloning state is [30]

$$
\Delta_{E P R}=V_{\text {out }}\left(x_{+ \text {clon }}\right)+V_{\text {out }}\left(p_{- \text {clon }}\right)
$$

The Wigner function of cloning state can be read as

$$
W_{\text {eprclon }}\left(\alpha_{1}, \alpha_{11}\right)=W_{+}\left(\alpha_{+ \text {clon }}\right) \cdot W_{-}\left(\alpha_{- \text {clon }}\right)
$$

The fidelity of the cloning state [27] is

$$
F=\pi \iint W_{\text {epr }}\left(\alpha_{1}, \alpha_{11}\right) W_{\text {eprclon }}\left(\alpha_{1}, \alpha_{1} 1\right) d^{2} \alpha_{1} d^{2} \alpha_{11}
$$


The overall the probability of success of the cloning of EPR state is the minimum between $P_{+}$and $P_{-}$

$$
P=\min \left\{P_{+}, P_{-}\right\}
$$

The results of numerical calculations are illustrated in Figure 4. Figure 4(a), 4(b) and 4(c) show the dependence of entanglement of the output entangled states $\Delta_{E P R}^{o u t}$, the fidelity $F$ and the successful cloning probability $P$ on the selection threshold respectively. The three entanglement degrees $(2.2 \mathrm{~dB}, 5.2 \mathrm{~dB}$, $10 \mathrm{~dB}$ ) of the input entangled states are shown in the figures. The entanglement of the cloned states increases with increasing of entanglement of input states; and the smaller the $Q$ is, the better the entanglement of cloned state is. Especially, with a coherent states input $\left(\Delta_{E P R}^{i n}=0.5\right)$, the output cloned states can only be coherent states without any entanglement (see orange dash dot dot line in Figure 4(a)). In other words, we can not produce entangled states with a coherent states input by the process. Because the entanglement is fragile and higher entanglement decays more easily, we can see in Figure 4(b) that the fidelity of cloned states increases with the decreasing of entanglement of the input states. For the $10 \mathrm{~dB}$ entangled states, the entanglement is almost lost and overwhelmed by vacuum noises, so it is difficult to purify the entanglement by the conditional selection. As a result, the fidelity value has a flat response with respect to $Q$ and remains below the $4 / 9$ limit for all $Q$.

For an input entanglement, it holds that the lower the threshold is, the better the fidelity is, however it is inevitable to sacrifice the probability of success $P$ (see in Figure 4(c)). The cloning efficiencies (fidelity and entanglement) are different for input states with different entanglement degrees, but they are all better than general cloning, so in a sense, it is effective for all entangled states.

The conditional selection process can also be understood in the way of harmonic mean state distillation [32], which means that one only keeps the outcome which is close to the input state, and the other outcomes are discarded, that is, the cloned states are kept from extra noise disturbing the cloning process. The cloning states are able to keep entanglement with fidelity even beyond the $4 / 9$ limit, when $Q$ is suitably selected, since in Figures 4(a) and 4(b) values for $Q$ can be found for which $\Delta_{E P R}>0.5$ and $F>4 / 9$. From the figures, we also can find that when the $Q$ is above 2 , which is 8 times normalized to the shot noise limit, the conditional cloning machine tends towards the general cloning machine [20].

\section{CONCLUSIONS}

In this article, a CV conditional cloning machine is proposed for the squeezed state and continuous-variable entangled states. The conditional cloning machine is a hybrid proposal which combines the continuous-variable and the conditional technique. Compared to the general CV cloning machine, some extra noise is suppressed and the clones with higher fidelity and entanglement are able to be obtained by comparing measured quadrature fluctuations with a threshold and taking effective feedback, but it is inevitable to sacrifice the probability of success. The lower the threshold is, the
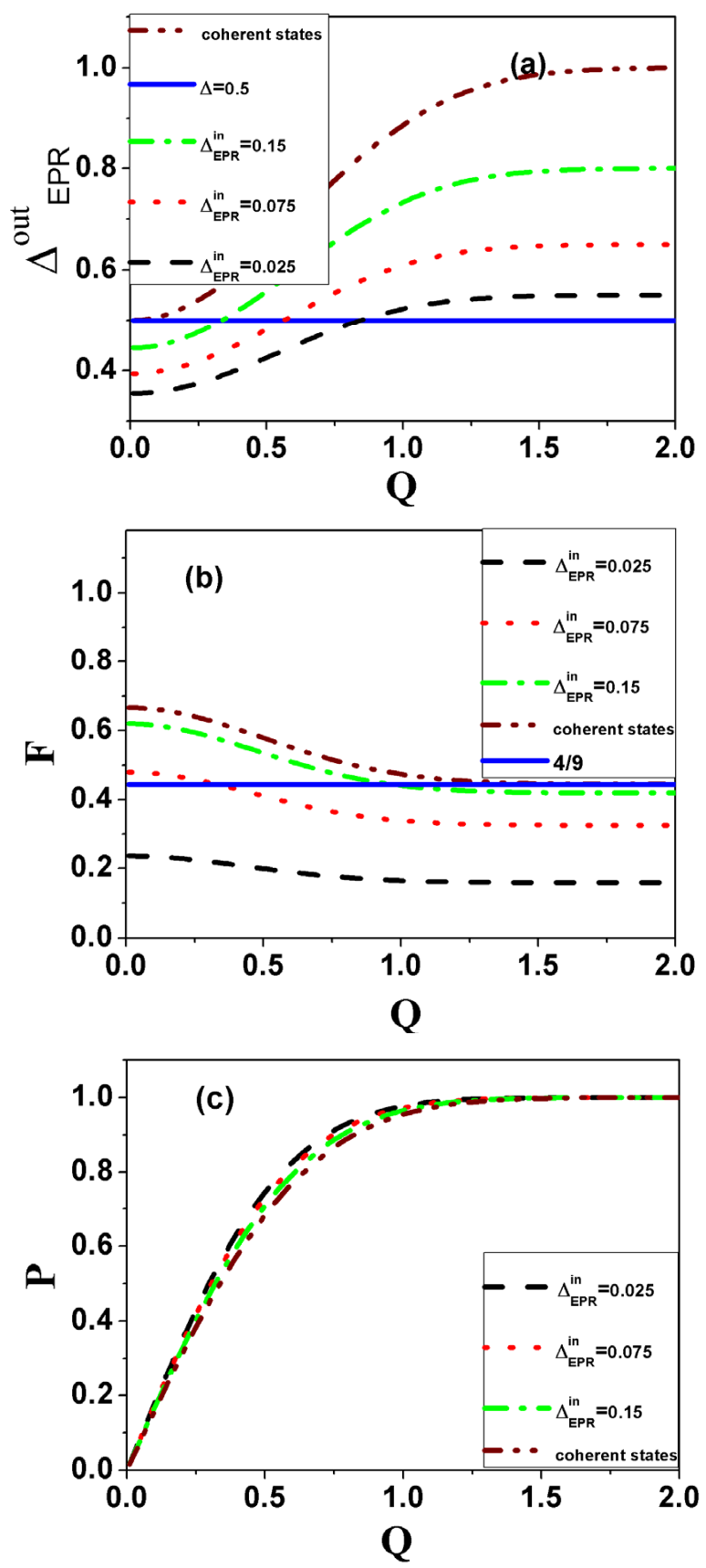

FIG. 4 (Color online) Results of conditional cloning depending on threshold value Q. (a) The entanglement variance of cloned entangled state $\Delta_{E P R}$; (b) the fidelity between the input and output states; (c) the probability of success. We consider three input states with different degree of entanglement. Coherent states (brown dash dot dot line ), $\Delta_{E P R}^{i n}=0.15$, corresponding to $2.2 \mathrm{~dB}$ entanglement (green dash dot line), $\Delta_{E P R}^{i n}=0.075$, corresponding to $5.2 \mathrm{~dB}$ entanglement (red dot line), $\Delta_{E P R}^{i n}=0.025$, corresponding to $10 \mathrm{~dB}$ entanglement (black dash line), shot noise limitation $\Delta=0.5$ (blue solid line in (a)). Optimal fidelity of entangled states cloning $4 / 9$ (blue solid line in (b)).

higher the fidelity and entanglement is. On the other hand, because the entanglement is fragile and a higher entanglement decays more easily, the effect of conditional selection becomes less obvious with increase of entanglement of input state and the result of fidelity has a flat response with respect to the threshold $Q$. In a sense, this cloning machine is controllable and practicable under the present experimental conditions, and has applications in quantum computation and quantum eavesdropping. Moreover, it can also be used as a probabilis- 
tically clone [33] with approximate perfect fidelity, and will be important for quantum identification and quantum deleting.

\section{ACKN OWLEDGEMENTS}

This work was supported by the National Natural Science Foundation of China (Grant Nos. 11274212, 61405108). National Natural Science Foundation of China Project for Excellent Research Team (Grant No. 61121064).

\section{References}

[1] W. K. Wooters, and W. H. Zurek, "A single quantum cannot be cloned," Nature 299, 802-803 (1982).

[2] V. Bužek, and M. Hillery, "Quantum copying: beyond the nocloning theorem," Phys. Rev. A 54, 1844-1852 (1996).

[3] V. Bužek, and M. Hillery, "Universal optimal cloning of arbitrary quantum states: from qubits to quantum registers," Phys. Rev. Lett. 81, 5003-5006 (1998).

[4] N. J. Cerf, A. Ipe, and X. Rottenberg, "Cloning of continuous quantum variables," Phys. Rev. Lett. 85, 1754-1757 (2000).

[5] Z. Zhai, J. Guo, and J. R. Gao, "Generalization of continuousvariable quantum cloning with linear optics," Phys. Rev. A 73 , 052302 (2006).

[6] J. Fiurásek, "Optical implementation of continuous-variable quantum cloning machines," Phys. Rev. Lett. 86, 4942-4945 (2001).

[7] S. L. Braunstein, N. J. Cerf, S. Iblisdir, P. van Loock, and S. Massar, "Optimal cloning of coherent states with a linear amplifier and beam splitters," Phys. Rev. Lett. 86, 4938-4941 (2001).

[8] N. J. Cerf, 0. Krüger, P. Navez, R. F. Werner, and M. M. Wolf, "Non-Caussian cloning of quantum coherent states is optimal," Phys. Rev. Lett. 95, 070501 (2005).

[9] S. Olivares, M. G. A. Paris, and U. L. Andersen, “Cloning of Gaussian states by linear optics," Phys. Rev. A 73, 062330 (2006).

[10] U. L. Andersen, V. Josse, and G. Leuchs, "Unconditional quantum cloning of coherent states with linear optics," Phys. Rev. Lett. 94, 240503 (2005).

[11] M. Sabuncu, U. L. Andersen, and G. Leuchs, "Experimental demonstration of continuous variable cloning with phase-conjugate inputs," Phys. Rev. Lett. 98, 170503 (2007).

[12] A. Einstein, B. Podolsky, and N. Rosen, "Can quantum-mechanical description of physical reality be considered complete?," Phys. Rev. 47, 777-780 (1935).

[13] Z. Y. Ou, S. F. Pereira, H. J. Kimble, and K. C. Peng, "Realization of the Einstein-Podolsky-Rosen paradox for continuous variables," Phys. Rev. Lett. 68, 3663-3666 (1992).

[14] M. D. Reid, and P. D. Drummond, "Quantum correlations of phase in nondegenerate parametric oscillation," Phys. Rev. Lett. 60, 2731-2733 (1988).

[15] A. Furusawa, J. L. Sørensen, S. L. Braunstein, C. A. Fuchs, H. J. Kimble, and E. S. Polzik, "Unconditional quantum teleportation," Science 282, 706-709 (1998).

[16] J. Jing, J. Zhang, Y. Yan, F. Zhao, C. D. Xie, and K. C. Peng, “Experimental demonstration of tripartite entanglement and controlled dense coding for continuous variables," Phys. Rev. Lett. 90, 167903 (2003).
[17] C. Silberhorn, N. Korolkova, and G. Leuchs, "Quantum key distribution with bright entangled beams," Phys. Rev. Lett. 88, 167902 (2002).

[18] N. C. Menicucci, P. van Loock, M. Gu, C. Weedbrook, T. C. Ralph, and M. A. Nielsen, "Universal quantum computation with continuous-variable cluster states," Phys. Rev. Lett. 97, 110501 (2006).

[19] C. Weedbrook, A. M. Lance, W. P. Bowen, T. Symul, T. C. Ralph, and P. K. Lam, "Coherent-state quantum key distribution without random basis switching," Phys. Rev. A 73, 022316 (2006).

[20] C. Weedbrook, N. B. Grosse, T. Symul, P. K. Lam, and T. C. Ralph, "Quantum cloning of continuous-variable entangled states," Phys. Rev. A 77, 052313 (2008).

[21] J. Laurat, T. Coudreau, N. Treps, A. Maître, and C. Fabre, "Conditional preparation of a quantum state in the continuous variable regime: generation of a sub-Poissonian state from twin beams," Phys. Rev. Lett. 91, 213601 (2003).

[22] J. Laurat, T. Coudreau, N. Treps, A. Maître and C. Fabre, "Conditional preparation of a nonclassical state in the continuousvariable regime: theoretical study," Phys. Rev. A 69, 033808 (2004).

[23] A. Franzen, B. Hage, J. DiGuglielmo, J. Fiurášek, and R. Schnabel, "Experimental demonstration of continuous variable purification of squeezed states," Phys. Rev. Lett. 97, 150505 (2006).

[24] B. Hage, A. Samblowski, J. DiGuglielmo, A. Franzen, J. Fiurášek, and R. Schnabel, "Preparation of distilled and purified continuousvariable entangled states," Nat. Phys. 4, 915-918 (2008).

[25] R. Dong, M. Lassen, J. Heersink, C. Marquardt, R. Filip, G. Leuchs, and U. L. Andersen, "Experimentally entanglement distillation of mesoscopic quantum states," Nat. Phys. 4, 919-923 (2008).

[26] H. Takahashi, J. S. Neergaard-Nielsen, M. Takeuchi, M. Takeoka, K. Hayasaka, A. Furusawa, and M. Sasaki, "Entanglement distillation from Gaussian input states," Nat. Photonics 4, 178-181 (2010).

[27] R. Jozsa, "Fidelity for mixed quantum states," J. Mod. Optic. 41, 2315-2323 (1994).

[28] H. Zhang, W. Liang, K. Liu, J. X. Zhang, and J. R. Gao, “Fidelity with quadrature component variances for continuous variable quantum teleportation," J. Phys. B 45, 115501 (2012).

[29] S. L. Braunstein, and H. J. Kimble, "Teleportation of continuous quantum variables," Phys. Rev. Lett. 80, 869-872 (1998).

[30] L. M. Duan, G. Giedke, J. I. Cirac, and P. Zoller, "Inseparability criterion for continuous variable systems," Phys. Rev. Lett. 84, 2722-2725 (2000).

[31] J. Fiurášek, P. Marek, R. Filip, and R. Schnabel, "Experimentally feasible purification of continuous-variable entanglement," Phys. Rev. A 75, 050302 (2007).

[32] M. Lassen, L. S. Madsen, M. Sabuncu, R. Filip, and U. L. Andersen, "Experimental demonstration of squeezed-state quantum averaging," Phys. Rev. A 82, 021801(R) (2010).

[33] L. M. Duan, and G. C. Guo, "Probabilistic cloning and identification of linearly independent quantum states," Phys. Rev. Lett. 80, 4999-5002 (1998). 